\title{
Perspective
}

PERSPECTIVE Actualité en histoire de l'art

1 | 2021

Portugal et espaces lusophones

\section{Cinéma, critique, arts visuels : autour de José Ernesto de Sousa (1921-1988)}

Un débat entre Tiago Afonso, António Contador, Sabrina D. Marques, Isabel Nogueira et Mickaël Robert-Gonçalves, mené par Raquel Schefer

Tiago Afonso, António Contador, Sabrina D. Marques, Isabel Nogueira, Mickaël Robert-Gonçalves et Raquel Schefer

Traducteur : Thomas Resendes

\section{(2) OpenEdition}

Journals

Édition électronique

URL : https://journals.openedition.org/perspective/22575

DOI : $10.4000 /$ perspective. 22575

ISSN : 2269-7721

Éditeur

Institut national d'histoire de l'art

Édition imprimée

Date de publication : 14 octobre 2021

Pagination : 61-78

ISBN : 978-2-917902-91-2

ISSN : $1777-7852$

Référence électronique

Tiago Afonso, António Contador, Sabrina D. Marques, Isabel Nogueira, Mickaël Robert-Gonçalves et Raquel Schefer, «Cinéma, critique, arts visuels : autour de José Ernesto de Sousa (1921-1988) »,

Perspective [En ligne], 1 | 2021, mis en ligne le 31 décembre 2021, consulté le 13 mai 2022. URL

http://journals.openedition.org/perspective/22575; DOI : https://doi.org/10.4000/perspective.22575 


\title{
Cinéma, critique, arts visuels : autour de José Ernesto de Sousa (1921-1988)
}

\author{
Un débat entre Tiago Afonso, António Contador, \\ Sabrina D. Marques, Isabel Nogueira et Mickaël \\ Robert-Gonçalves, mené par Raquel Schefer
}

José Ernesto de Sousa aurait eu cent ans en avril 2021. Né à Lisbonne pendant la Première République (1910-1926), en 1921 , année de la fondation du Parti communiste portugais et de la " nuit sanglante " (Noite Sangrenta) du 30 septembre - le coup d'État qui préfigure celui de 1926 et les quarante-huit ans de régime fasciste et colonial -, Ernesto de Sousa est l'une des figures les plus complexes et prolifiques de son temps. Artiste interdisciplinaire, essayiste et commissaire d'exposition, opérant dans les champs des arts plastiques, du théâtre, de la photographie et du cinéma, il a aussi mené un important travail pédagogique et critique. Si dans la vie et l'œuvre d'Ernesto de Sousa se nouent la fonction sociale (à travers son opposition politique et culturelle au régime, son rôle fondamental dans la formation des publics, celui d'un observateur libre et critique) et la dimension expérimentale de l'art, cet artiste-producteur incontournable contribue également à relier, tantôt au niveau de sa pratique exemplaire, tantôt selon une perspective critique et historiographique, les différents mouvements artistiques du $\mathrm{XX}^{\mathrm{e}}$ siècle - en particulier, les modernismes - au Portugal. Ce geste crucial se fonde sur une logique de continuité et de rupture. Ainsi, débutant sa carrière dans les années 1940 sur le terrain du néoréalisme, Ernesto de Sousa non seulement concrétise une révision critique du premier modernisme portugais - notamment de l'œuvre d'Almada Negreiros, à qui il consacre, entre 1969 et 1972, le film " élargi ${ }^{1}$ " Almada, um nome de guerra, ainsi que du travail d'Eduardo Viana et de Santa-Rita Pintor -, mais jette aussi des ponts entre les différentes générations artistiques au Portugal et entre celles-ci et la néo-avant-garde internationale, notamment le mouvement Fluxus. Comme le souligne l'historienne de l'art Isabel Nogueira, qui participe à cette discussion, en 1972, à son retour de la " Documenta 5 " à Cassel, Ernesto de Sousa déclenche " un processus de réflexion sur l'art portugais du moment ${ }^{2}$ ", processus qui culmine, cinq ans plus tard, avec le commissariat de l'exposition la plus paradigmatique des années 1970 au Portugal, " Alternativa zero: tendências polémicas na arte portuguesa contemporânea ", à la Galeria nacional de arte moderna à Lisbonne. Trois ans après la Révolution de 1974-1975, cette exposition singulière, qui réunit des " opérateurs esthétiques" ${ }^{3}$ " (Alberto Carneiro, Ana Hatherly, Fernando Calhau et Helena Almeida, entre autres), signale, aux prismes 
matériel et symbolique, le dialogue esthétique et politique de l'art portugais avec la scène internationale, tout en questionnant la proposition selon laquelle il appartiendrait aux " pays centraux " de produire les expériences artistiques avant-gardistes.

À propos d'Almada Negreiros, figure tutélaire du premier modernisme portugais qu'il rencontre en 1946, lors de l'organisation, avec Diogo de Macedo, d'une exposition d'art moderne et d'art africain à Lisbonne explorant, dans la lignée des avant-gardes historiques, les relations entre modernisme et primitivisme, Ernesto de Sousa écrit : " Le fait qu'il soit peut-être l'homme le plus extraordinaire que j'aie jamais rencontré ne fait pas beaucoup avancer l'affaire. Mais en le nommant, je découvre un NOM DE GUERRE [sic] pour tout ou presque tout ce qui m'intéresse le plus ${ }^{4}$ ". Ce " nom de guerre " permet à Ernesto de Sousa de placer sa propre production artistique - aussi bien que sa réflexion théorique et son travail en tant que commissaire d'exposition - dans le prolongement du premier modernisme portugais. Ses propositions pour une nouvelle avant-garde portugaise s'inscrivent dès lors dans cette généalogie. José Ernesto de Sousa, un autre nom de guerre pour les générations à venir, considère, comme Nogueira le souligne, qu'il n'existe qu'une seule avant-garde ${ }^{5}$, cosmopolite et dynamique, située entre la tradition et l'invention, ouverte sur le passé et vers l'avenir.

La définition et la praxis de l'" avant-garde " d'Ernesto de Sousa traversent cette discussion. Si Nogueira considère que, pour Ernesto de Sousa, l'avant-garde visait " la globalisation, la découverte de nouvelles structures, la participation, l'action ", la critique et cinéaste Sabrina D. Marques souligne que l'artiste la définit comme " un outil analytique, un processus ouvert ". L'artiste et théoricien de l'art António Contador remarque quant à lui que l'art et la vie impliquent, pour Ernesto de Sousa, l'« opérationnalisation du changement ", alors que le cinéaste Tiago Afonso affirme que " tout grand créateur est un avant-gardiste esthétique dans le champ politique et un avant-gardiste politique dans le champ de l'art, ou de l'esthétique ".

L'intersection entre esthétique et politique - et entre art et " praxis vitale ${ }^{6}$ " - structure la vie et l'œuvre d'Ernesto de Sousa. Parmi ses multiples pratiques et fronts d'action, il est important de noter ses activités dans le champ du ciné-clubisme. En 1946, Ernesto de Sousa fonde le Círculo do Cinema (" cercle du Cinéma "), l’un des premiers cinéclubs au Portugal, dont l'interdiction par la PIDE (Police internationale et de défense de l'État), la police politique du régime, deux ans plus tard, entraîne la détention de l'artiste. Le ciné-clubisme est alors conçu comme une pratique de résistance esthétique et politique, notamment à travers la diffusion de films expérimentaux et la réalisation d'ateliers de cinéma expérimental, comme celui qui eut lieu à Porto en 1965. Selon l'historien du cinéma Mickaël Robert-Gonçalves, lorsqu'il fonde la Cooperativa do Espectador en 1959, Ernesto de Sousa « est [...] le premier à (ré)affirmer la dimension collective de la création cinématographique qui a été, quelques années après, au cœur du moment collectif du cinéma portugais ". Dom Roberto (1962), premier et seul long métrage d'Ernesto de Sousa, produit au sein de cette structure, démontre que, pour l'artiste, la révolution des formes est inséparable de la révolution des modes de production. Ce film, qui opère des passages entre le néoréalisme et le Novo Cinema portugais, et qui contribue à forger le langage de ce dernier, reçoit deux prix au festival de Cannes en 1963. Détenu par la PIDE à nouveau, Ernesto de Sousa est empêché d'assister au festival.

L'œuvre d'Ernesto de Sousa est traversée par une impulsion collectiviste liée notamment à l'imprégnation du tissu filmique par la culture populaire, aussi bien qu'à ses modes de production collectifs. Dans les séries photographiques Affiches politiques et Graffitis politiques (vers

1. Ernesto de Sousa, deux photographies issues de la série Affiches politiques, 1974-1975, Centro de estudos multidisciplinares Ernesto de Sousa (CEMES). 

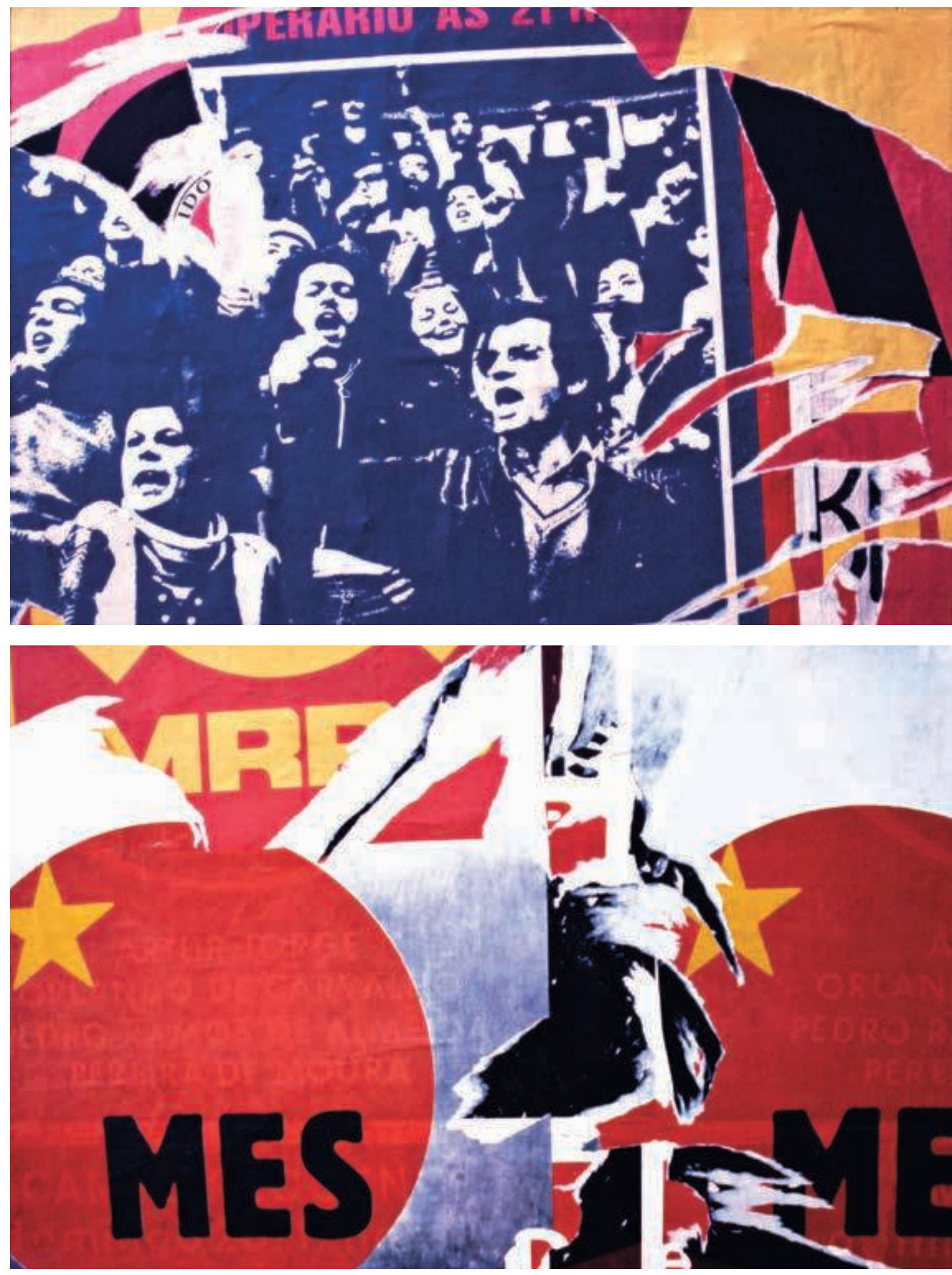
1974-1975 ; fig. la-b), l'artiste documente la révolution de 1974-1975. Ces deux séries établissent un dialogue thématique et formel fécond avec le film Revolução (Révolution, 1975), d'Ana Hatherly, un travail expérimental, tourné en super-huit, dont le montage assemble une série de très gros plans de fresques murales, de graffitis et d'affiches de la période révolutionnaire. Ces ouvres font partie d'un corpus qui transforme le fond et la forme de l'art consacré à la révolution, articulant inventivité esthétique et engagement politique, faisant écho à la position d'Ernesto de Sousa à l'égard de la fonction de la production esthétique dans les contextes révolutionnaires. Pour l'artiste, les avant-gardes esthétiques, expressions d'une pratique artistique et culturelle autonome, sont nécessaires dans de tels contextes. Dans son ouvre, l'impulsion collectiviste acquiert encore une autre dimension, liée aux méthodologies et aux modes opératoires mis en œuvre, remet en cause tout un ensemble de catégories esthétiques modernes, telles celles d'« auteur " et d'« œuvre d'art ". Almada, um nome de guerra (techniques mixtes, cinéma, arts graphiques, poésie, musique et peinture), par exemple, avait été conçu pour être projeté simultanément sur sept écrans. Cette forme qui s'inscrit dans une généalogie remontant au Napoléon (1927) d'Abel Gance ainsi qu'à certaines expériences développées notamment par Edgar Reitz et Stan Vanderbeek, vise à créer un public participant. La production du film est accompagnée de l'écriture du manifeste inédit " Anticinema ${ }^{7}$ ". Dans cette réflexion sur le film dédié à Almada Negreiros, le lecteur est incité à compléter les phrases et la ponctuation. Cet appel à une lecture collaborative - " opérative " selon les mots d'Ernesto de Sousa - synthétise sa conception collectiviste de la production artistique. Ernesto de Sousa invite le lecteur / spectateur à " une autre forme de travail collectif ", à " un art collectif, un art de la participation ", un " art ouvert ou un anti-art ", avec le but de " détruire reconstruire ?) [sic] le cinéma ${ }^{8}$ ". Le cinéma devient donc un processus de cocréation et d'échange, ce qui remet en question l'extériorité de l'objet filmique pour le destinataire, ouvrant la possibilité d'un décentrement des perspectives, en lien avec les expériences mondiales de collectivisation de la production cinématographique de cette période historique. Cette conception collectiviste et processuelle s'accorde avec les propos d'Ernesto de Sousa à l'égard de l'exposition "Alternativa zero ", qui vise, selon ses termes, à " démontrer l'importance mineure de l'objet d'art par rapport aux sujets impliqués dans l'activité esthétique, par rapport au PROCESSUS ESTHÉTIQUE [sic] ${ }^{9}$ ".

Tous ces aspects font de José Ernesto de Sousa l'un des artistes et l'un des penseurs de l'art les plus importants de son temps au Portugal et au niveau international. À l'heure où son héritage est réévalué, comme le démontre la récente production académique et les événements sur le sujet, tel le colloque " Ernesto de Sousa " qui eut lieu à la fondation Calouste Gulbenkian, à Lisbonne, en juin 2021, cette discussion collective cherche à contribuer à une connaissance plus ample de sa vie et de son ouvre en France. [Raquel Schefer]

- Raquel Schefer. À la lumière de vos travaux et tenant compte du parcours théorico-pratique interdisciplinaire de José Ernesto de Sousa - son action dans différents domaines (production artistique, cinématographique et littéraire, commissariat d'exposition, ciné-club, critique et pédagogie, etc.) - quelle place donneriez-vous à la figure de l'artiste dans le panorama des arts portugais de la seconde moitié du XXe siècle?

- Isabel Nogueira. José Ernesto de Sousa est une figure incontournable de l'art portugais des années 1970 et 1980. Dès 1969, il organise le Meeting as Art Encontro no Guincho ${ }^{10}$ 
(fig. 2) et la performance mixte Nós não estamos algures au club de théâtre Primeiro Acto (Algés), s'inspirant de la conférence "A invenção do dia claro » (1921) d'Almada Negreiros. Ces événements sans précédent au Portugal permettent d'expérimenter l'espace de la rencontre comme forme d'art. Tour à tour critique, cinéaste, " opérateur esthétique », journaliste, essayiste, commissaire d'exposition, enseignant, membre de cinéclubs, Ernesto de Sousa était un catalyseur reliant des personnes et des pratiques artistiques, malgré un parcours éclectique et relativement fragmenté. On peut souligner, entre autres, son activité de commissaire d'exposition, dès $1972^{11}$. La même année, il se rend à Cassel pour la manifestation historique "Documenta 5 », dirigée par Harald Szeemann, et publie un entretien avec Joseph Beuys dans le journal República ${ }^{12}$. Mais c'est seulement en 1977 qu'il organise l'exposition collective la plus importante de la décennie : «Alternativa zero: tendências polémicas na arte portuguesa contemporânea ${ }^{13}$ ", représentant le travail d'un groupe néo-avant-gardiste effervescent, dans un pays récemment sorti de la dictature. Regroupant une cinquantaine de participants, cette exposition marque à la fois
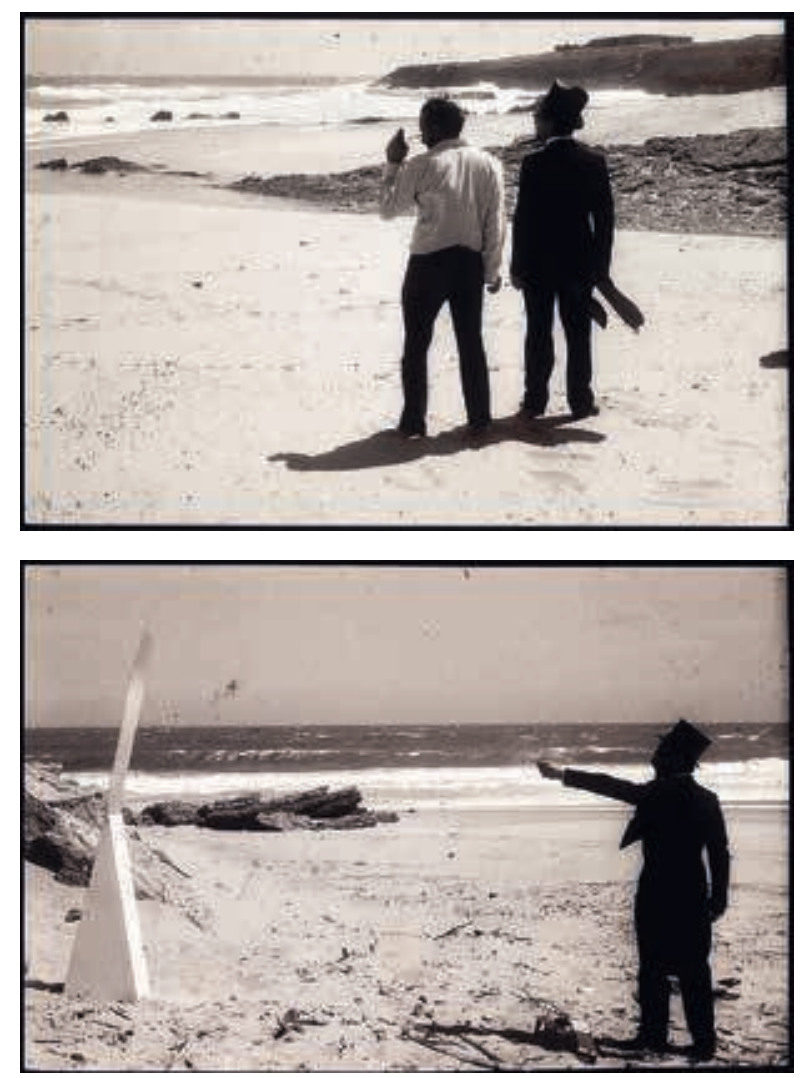

2. Sur la plage de Guincho, le 3 avril 1969, à l'occasion de la rencontre organisée par Ernesto de Sousa en collaboration avec Noronha da Costa et l'Oficina experimental, CEMES.

l'apogée du mouvement néo-avant-gardiste et les prémices du courant post-moderne, caractérisé par l'usage de la citation, de l'ironie et par le caractère référentiel de certains travaux. Indépendamment des formats, des supports ou des concepts, c'est avant tout la recherche d'une relation entre l'art et la vie et le rapport à la modernité qui ont marqué le parcours exemplaire d'Ernesto de Sousa. En d'autres termes, cet artiste incarne une profonde urgence d'être moderne ${ }^{14}$.

- Mickaël Robert-Gonçalves. Dans le cadre de mes recherches doctorales sur le cinéma portugais en Révolution (1974-1982) ${ }^{15}$, le nom d'Ernesto de Sousa est d'abord apparu lors de l'étude du mouvement des ciné-clubs au Portugal au début des années 1950, puis à la sortie du film Dom Roberto en 1962 (fig. 3). Grâce à l'influence des ciné-clubs, dont il demeure le pionnier au Portugal avec notamment le Círculo do Cinema créé en 1946, il a été le passeur de toute une génération. Lors de notre entrevue en 2011, le cinéaste Alberto Seixas Santos a souligné le rôle des ciné-clubs au Portugal à cette époque :

Ce mouvement a eu une grande influence, indiscutablement. C'est grâce à cela que nous avons appris à voir le cinéma. La suite logique, c'est que nous avons fini par être dirigeants de ciné-clubs, incitant nos collègues étudiants à rejoindre ce mouvement culturel de premier plan. À mon avis, au Portugal, c'est l'élément le plus important pour comprendre la suite ${ }^{16}$. 


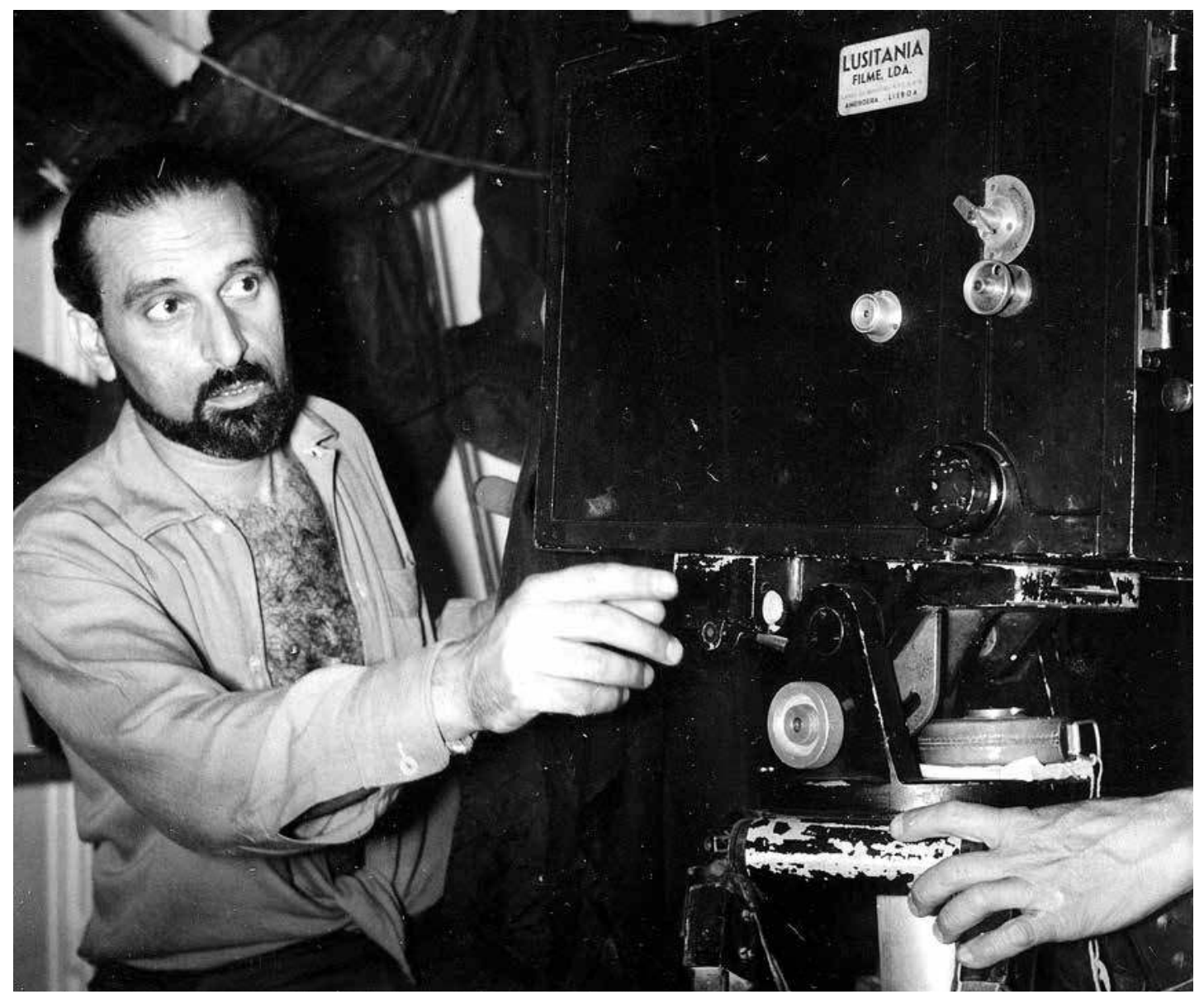

3. Ernesto de Sousa pendant le tournage

En ayant l'idée d'une coopérative ${ }^{17}$, il est le premier de Dom Roberto, 1962, CEMES.

à (ré)affirmer la dimension collective de la création cinématographique qui, quelques années plus tard, a été un élément fondamental du cinéma portugais que j'ai longuement étudié dans ma thèse. Enfin, durant les années 1970, son implication critique et sa pratique interdisciplinaire ont, selon moi, permis d'attirer les regards sur de nouveaux espaces d'expression au Portugal - ce faisant, l'art portugais s'est inscrit plus fortement dans l'ère post-moderne. En cela, Ernesto de Sousa doit être considéré comme un des artistes portugais les plus féconds de la seconde moitié du XXe siècle.

- Tiago Afonso. Ernesto de Sousa a exercé une influence relativement tardive sur mon travail. Je préfère parler de consonances ou de parallèles. Nous avons une influence majeure en commun : I'incontournable Mário Dionísio, dont j'ai étudié le travail et avec qui Ernesto de Sousa dialoguait. Ils avaient tous les deux l'ambition d'atteindre le grand public, de s'adresser à tous. Pour ma part, j'essaie de faire des films qui ne soient pas hermétiques, mais importants, justes et accessibles, même s'ils contiennent plusieurs niveaux de lecture. Comme c'était déjà le cas à l'étranger, Ernesto de Sousa a créé un commissariat d'exposition horizontal au Portugal, entremêlant primitivisme et art moderne. Il a exposé sur un même plan des 
artisans de Barcelos et des auteurs de l'élite moderniste portugaise (fig. 4a-b). Il a également été l'un des premiers à défendre l'idée que la peinture devait sortir des tableaux et investir les murs publics (les fresques). On a suspecté le film Dom Roberto, qui se rattache autant au néoréalisme italien qu'aux premières œuvres des différentes nouvelles vagues dans le monde, d'être populiste, en raison du choix de Raúl Solnado pour incarner le rôle principal. On l'a accusé d'être en deçà du nouveau cinéma portugais, en termes d'objectifs, avec ce film... Lorsqu'il a été sélectionné au festival de Cannes, la PIDE a retenu Ernesto de Sousa à l'aéroport. Finalement, cette résistance formelle et politique, son éclectisme manifeste et son refus de distinguer entre la forme et le contenu m'ont beaucoup aidé à conserver certaines « certitudes » formelles et déontologiques. Je pense particulièrement au travail avec les communautés les plus fragiles et à certaines thématiques émancipatrices.

L'importance d'Ernesto de Sousa s'est accrue dans la seconde moitié du XXe siècle, grâce à la diffusion et à l'étude de son œuvre d'auteur, de critique, de conservateur et de pédagogue. Le Portugal a reconnu très tardivement sa véritable influence (Dom Roberto demeure une merveille cinématographique encore aujourd'hui méconnue).

- Sabrina D. Marques. Et si le nom d'Ernesto de Sousa était synonyme du mot révolution ? Pour le XXe siècle portugais, son action est sans précédent. Sur un plan pratique et théorique, il pose les bases d'une refondation culturelle et artistique au Portugal, émancipant les normes esthétiques du pays des modèles hermétiques du fascisme. Son insatiable préoccupation s'inscrit dans un héritage vaste et multiforme qui, comme nul autre auparavant, a ouvert sur le monde un pays jusqu'ici replié sur lui-même. Sans la moindre condescendance ou prétention pédagogique, on peut dire que son travail aura des répercussions formatrices pour des générations de chercheurs et d'artistes qui poursuivront ses travaux jusqu'à nos jours.

- Raquel Schefer. L'influence de l'avant-garde internationale d'après-guerre sur l'œuvre d'Ernesto de Sousa est étudiée sur un plan théorique et critique. Considérez-vous que son travail ait permis une révision critique de l'art portugais? De plus, compte tenu de la diffusion internationale de son œuvre, faut-il remettre en question le récit selon lequel les expériences artistiques d'avant-garde sont généralement produites au sein des pays centraux?

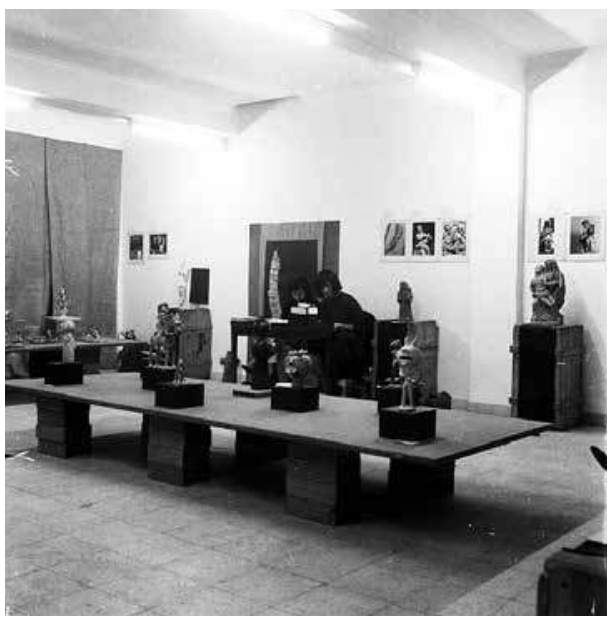

4a-b. Vues de l'exposition «Barristas e imaginários: quatro artistas populares do Norte », Lisbonne, Livraria Divulgação, mai-juin 1964 (avec des œuvres de Mistério, Rosa Ramalho, Quintino Vilas Boas Neto et Franklin Vilas Boas), CEMES.

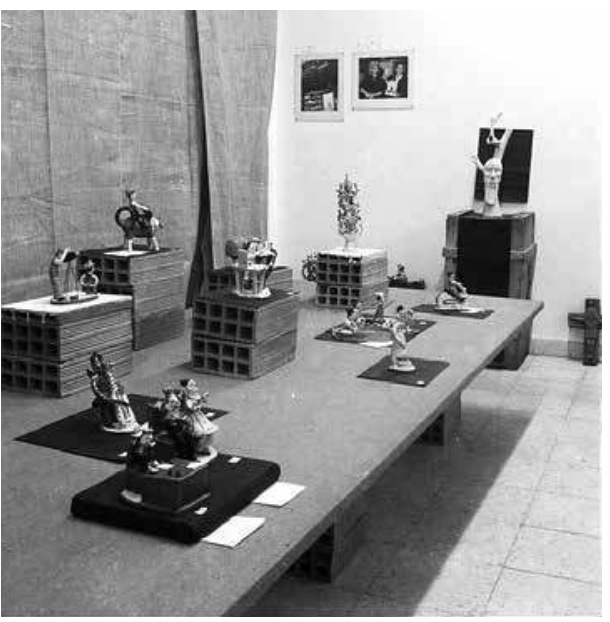


- Sabrina D. Marques. Ernesto de Sousa aurait découvert l'œuvre cinématographique et les performances d'Andy Warhol en 1969, lors d'une rétrospective au Rex Cinema de Londres consacrée à l'artiste nord-américain. Il aurait été stupéfait par le caractère avant-gardiste de ces projections offrant aux spectateurs une expérience libératrice, en leur permettant de vivre le cinéma à leur propre rythme, d'entrer et de sortir de la salle. Ernesto de Sousa n'a cessé de faire l'apologie de l'expérimentalisme comme pratique transversale, fondamentalement libre et dégagée des problématiques de nationalité, d'idéologie et de générations - ce qui ne l'a pas empêché de plébisciter certaines personnalités triées sur le volet parmi un vaste réseau d'influence. Sa relation avec Almada Negreiros, notamment, reste incompréhensible du point de vue de la jeunesse du Cinema novo. Ernesto de Sousa cite fréquemment cet " artiste contemporain incontournable ", notamment la phrase " une œuvre d'art ne doit être que commencée ». De fait, la notion d'esquisse est l'une des composantes les plus importantes de son œuvre. II a développé ce concept sous diverses formes, tout d'abord en considérant son carnet d'écriture comme un objet en soi, dans lequel chaque page de notes et chaque réunion importante sont des espaces sans commencement ni fin, soumis à une actualisation permanente. D'autre part, en structurant peu à peu la conceptualisation d'une notion essentielle, à savoir la notion de obra aberta (« œuvre ouverte »).

- Isabel Nogueira. Cette question soulève plusieurs problématiques, notamment celles de l'avant-garde et de la néo-avant-garde. À la fin des années 1960, Ernesto de Sousa commence à suivre de près le mouvement de l'avant-garde, de l'art conceptuel et plus largement du conceptualisme. Son intérêt pour le néoréalisme (rappelons que Dom Roberto est réalisé au sein de ce courant) se déplace vers ce qu'il appelle lui-même " l'avant-garde ». À ce sujet, s'il est fondamental de distinguer " l'avant-garde",

5. Vue de l'exposition « Alternativa zero: tendências polémicas na arte portuguesa contemporânea ", Lisbonne, Galeria nacional de arte moderna, février-mars 1977, CEMES. comme substantif au sens critique, du mouvement néo-avant-gardiste international (pop art, nouveau réalisme, art conceptuel, art minimal, arte povera,

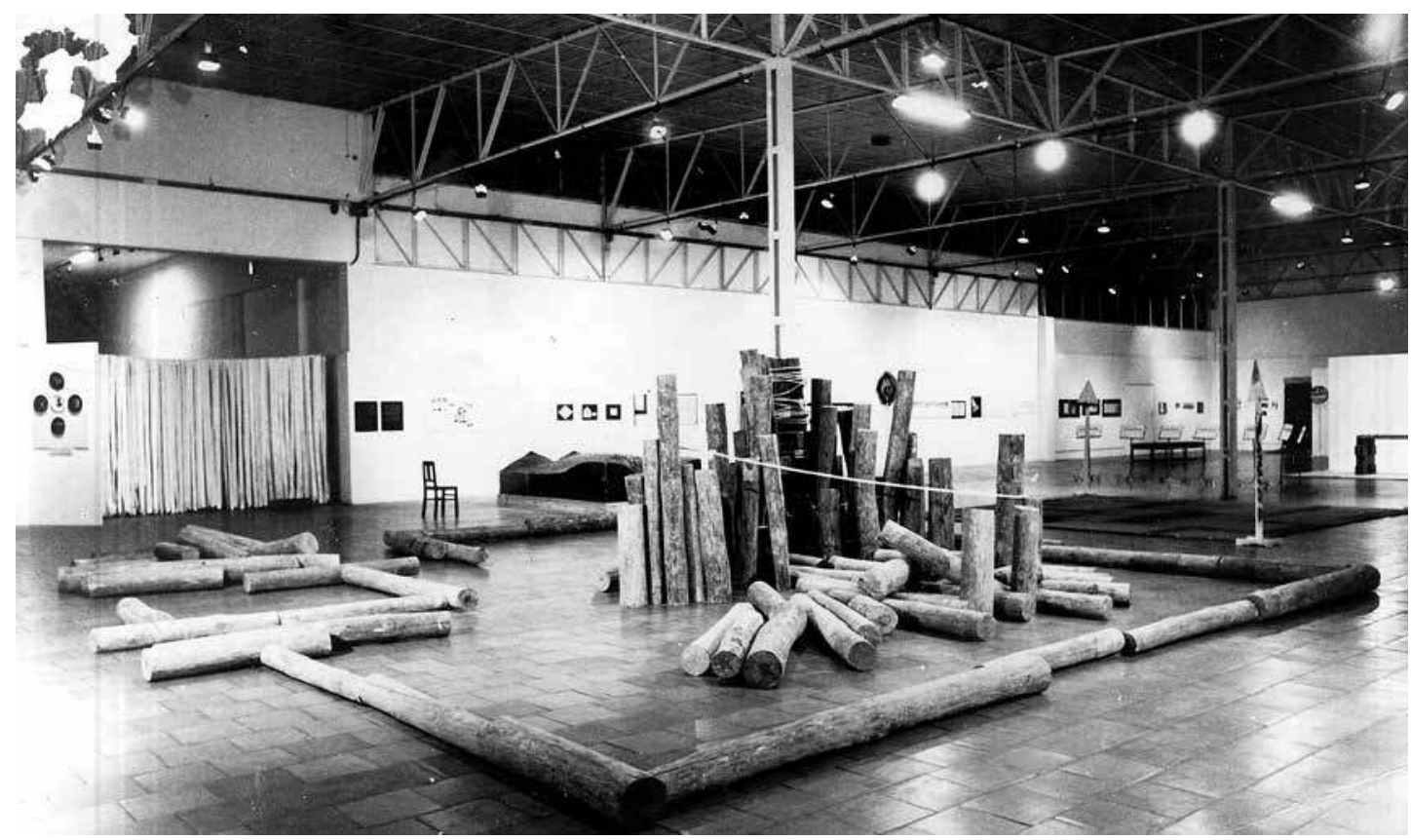


performativité, etc.), Ernesto de Sousa, quant à lui, ne distingue pas clairement ces deux notions. Dans un texte datant de 1977, il déclare « J'ai pu étudier scrupuleusement l'évolution des avant-gardes, ou plutôt de l'avant-garde, car il n'y en a qu'une ${ }^{18}$. » Ernesto de Sousa considérait le terme "d'avant-garde » comme une sorte de " métaphore militaire », nécessitant une " arrière-garde ", autrement dit, un passé ${ }^{19}$. La notion d'avant-garde impliquerait une coupure épistémologique indissociable d'un processus de mutation historique ${ }^{20}$. Autrement dit, l'action d'Ernesto de Sousa est essentiellement guidée par sa conscience de l'avant-garde et de la modernité, plus encore que par une connexion spécifique avec les mouvements néo-avant-gardistes internationaux.

Il faut, par ailleurs, distinguer la question des expériences artistiques de celle de la relocalisation de l'art produit au Portugal sur le plan international, et des divers mouvements artistiques (art conceptuel, art minimal, etc.). Les mouvements artistiques qui ont constitué la néo-avant-garde internationale ont eu lieu, de fait, dans des territoires dont le rayonnement culturel est important (France, Italie, Royaume-Uni, Allemagne ou États-Unis d'Amérique). Cela dit, les expériences artistiques portugaises des années 1970 (et des années 1980, puisque le Portugal a pris part au mouvement post-moderne, et qu'il en était même l'un des protagonistes) ont joué un rôle essentiel et novateur, sous la direction d'un groupe d'artistes remarquables pour lesquels l'action d'Ernesto de Sousa fut d'un grand poids.

- Tiago Afonso. Les expériences artistiques d'avant-garde n'ont pas de nationalité ou de territoires prédéfinis. En revanche, elles ont des langages (verbaux, esthétiques, plastiques, imaginaires, éthiques...), et sont reconnues, distribuées, diffusées, patrimonialisées dans des espaces particuliers et, à ce titre, référencées en tant que pratique propre à ces territoires. Dans le cas d'Ernesto de Sousa, déclaré persona non grata par le pouvoir jusqu'en 1974, l'internationalisation était la seule alternative.

Les préoccupations d'Ernesto de Sousa au début de la seconde moitié XXe siècle étaient inextricablement liées à d'autres problématiques soulevées à l'étranger, en ces temps où les mouvements d'opposition aux régimes totalitaires étaient fortement influencés par les théories marxistes et, plus tard, marxistes-léninistes. Le modèle à adopter était celui du « réalisme socialiste ", précédé d'une analyse matérialiste-dialectique. Par conséquent, Mário Dionísio, Ernesto de Sousa, Júlio Pomar, António Lopes Graça, parmi d'autres, comme Bertolt Brecht ou Walter Benjamin, étaient en rupture avec la vision réaliste de l'art prônée par le Parti communiste portugais (PCP) qu'ils jugeaient dogmatique. C'est pourquoi je suis d'accord pour dire que cet auteur a permis une véritable révision critique de l'art, dans ses formes de représentations et d'expressions...

- António Contador. Ernesto de Sousa avait une vision de l'exposition à contre-courant d'une certaine tradition du "salon », très en vogue au Portugal, durant ces années-là. Dans un papier sur l'exposition «Alternativa zero » (fig. 5) dont il est commissaire, il écrit :

LE SALON. La récurrence de termes français signale l'existence d'un certain style européen au sein de la culture portugaise. Les vernissages et autres manifestations ont toujours eu du succès - peut-être parce qu'ils bénéficient de la garantie d'un petit groupe de personnes, qui constituent précisément "le milieu", une micro-classe sociale relativement autonome, ayant ses propres ressources. Au Portugal, "nous sommes trois cents à prétendre être cultivés", selon l'expression truculente d'un écrivain des années 1940. Pour autant, les autres ne sont pas totalement exclus, dans la mesure où le milieu artistique leur offre le bénéfice d'un savoir lointain, mais prestigieux, succédané des rituels auliques, appréciés avec plus ou moins d'authenticité. [...] Pour résumer, cette hypocrisie dont le "salon" est un des grands symboles (le musée, généralement, en est un autre) peut être appréciée par le commun des mortels 


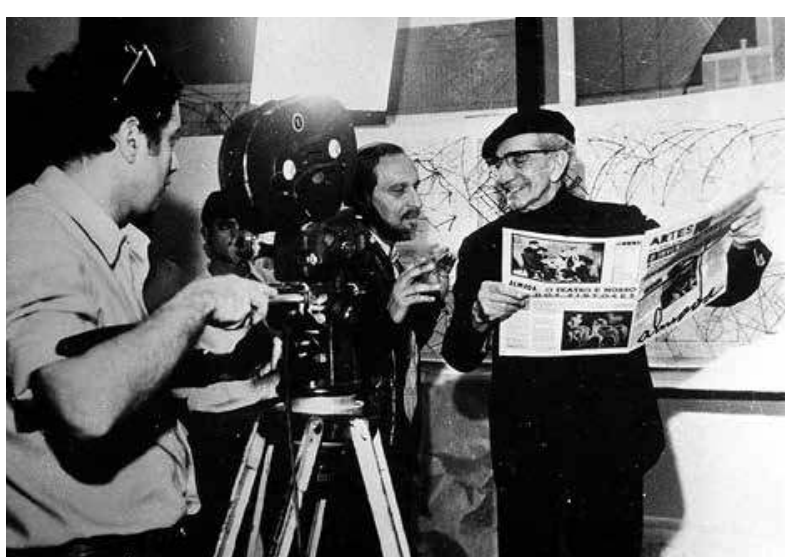

6a. Manuel Costa e Silva (à la caméra), Ernesto de Sousa et Almada Negreiros dans son atelier, pendant le tournage d'Almada, um nome de guerra, 1969, CEMES. de différentes façons, allant de la fatuité du détenteur à la fausse illusion de possession - une forme kitsch qui touche surtout les classes moyennes ${ }^{21}$.

À ce propos, Szeemann est décrit par Ernesto de Sousa comme un homme d'aplomb, capable de rompre médiatiquement et artistiquement avec la tradition du « salon ». La revue Chroniques de l'art vivant publie un article intéressant de la critique française Irmeline Lebeer ${ }^{22}$ qui, après avoir visité la "Documenta 5 », s'étonne de la rupture programmatique et paradigmatique opérée par Szeemann en 1972 - passant de l'image du musée comme d'un lieu foncièrement bourgeois, discriminant et exclusif, à celle d'un lieu de rencontre, dans lequel des alternatives artistiques et sociales sont discutées et débattues. Cet article s'ouvre sur une citation assez éclairante de Maurice Blanchot : "L'art n'est plus dans la "perfection" d'une œuvre, il n'est nulle part, et si le musée a un sens, c'est qu'il semble être ce "nulle part" dont il porte l'inquiétude et la puissante négation ${ }^{23}$.»

À mon sens, la « Documenta 5 » a incarné ce «nulle part » vibrant d'inquiétude et d'abnégation, aux yeux d'Ernesto de Sousa ${ }^{24}$, comme aucun autre lieu ou espace d'exposition. Son passage à Cassel lui démontre (ou, peut-être, n'est ce que la confirmation de son intuition première) que l'art, la vie, sont inintéressants sans cette mise à jour perpétuelle du changement et de la destruction de soi, levier puissant et non moins cruel de la liberté. Que peut-on faire, une fois qu'on a compris cela? Le partager?

- Raquel Schefer. Compte tenu des différents champs d'action d'Ernesto de Sousa, de son engagement politique et de son intérêt pour les modes de production, de diffusion et de réception artistiques, comment situeriez-vous l'œuvre de cet artiste par rapport aux notions « d'avant-garde esthétique » et « d'avant-garde politique » ?

- Tiago Afonso. Tout ce que je peux dire, c'est que tout grand créateur est un avant-gardiste esthétique dans le champ politique et un avant-gardiste politique dans le champ de l'art, ou de l'esthétique. C'est le cas d'Ernesto de Sousa.

- Mickaël Robert-Gonçalves. Mon analyse est sans doute toujours un peu biaisée par l'effusion révolutionnaire que j'ai étudiée - un moment de l'histoire de l'art durant lequel tout était politique, mais il me semble que le travail d'Ernesto de Sousa a souvent réussi à joindre « avant-garde esthétique » et « avant-garde politique ». Déjà avec Dom Roberto, il proposait un style novateur tout en s'appuyant sur le public. Néanmoins, sa sortie est apparue comme un essai ambigu et « déceptif », pour reprendre l'expression de la chercheuse Ilda Castro ${ }^{25}$, car le film ne rencontra pas son public. II s'agit pourtant du premier (et unique) film produit par le mouvement des ciné-clubs, suite à la création, en 1960, d’une coopérative spécifique par la revue Imagem. Précurseur de la démarche coopérative du cinéma portugais - qui sera omniprésente durant la révolution -, il a, par ce biais, toujours considéré l'art comme une source d'ouverture et de partage, même si le public ne le lui rend pas toujours. Il écrivait ainsi : 
Un des dénominateurs communs significatifs de l'avant-garde actuelle est la lutte contre une tendance élitaire de l'art fait par des spécialistes, destinée au divertissement passif de la majorité et à la possession égoïste de rares privilégiés. C'est une lutte pour une meilleure ou une totale participation de tous. C'est la praxis tirée de cette célèbre affirmation du grand précurseur Lautréamont : "La poésie doit être faite par tous. Non par un ${ }^{26 . "}$

- Isabel Nogueira. Ernesto de Sousa était un artiste, un critique et un théoricien. Pour répondre à votre question, je considère que son action en tant que commissaire d'exposition, critique et théorique présentait des éléments bien plus significatifs. Selon lui, la notion d'avant-garde impliquerait un dialogue entre les différentes formes d'avant-gardes, à savoir esthétique et idéologique. L'exposition " Alternativa zero » serait la transcription fidèle et innovatrice de l'expression "politique mais non partisane ». Toutes les avant-gardes convergeraient vers cette voie conceptuelle ${ }^{27}$. La vision avant-gardiste d'Ernesto de Sousa conduirait au rejet et à la destruction de tout objet esthétique s'éloignant d'une praxis vitale, qui serait élitiste, de l'ordre du prestige, du divertissement ou du spectaculaire indépendant de la vie elle-même. Ce serait l'expression de la lutte contre le consumérisme, le « kitsch » et l'accessible. Ce serait considérer l'art comme un work in progress, en valorisant l'éphémère et la simultanéité. L'avant-garde serait donc un processus de globalisation, de découverte de nouvelles structures, de participation, d'action. Elle utiliserait les techniques de la provocation et de l'humour, ainsi que toutes sortes de matériaux. Son désir serait celui d'une société socialiste, dans laquelle I'utopie se réaliserait au présent, par l'invention d'un nouveau rituel, un rituel festif28. Voilà donc la relation qu'Ernesto de Sousa a établie entre l'avant-garde et la politique. D'ailleurs, Ernesto de Sousa était un fervent admirateur de Joseph Beuys qu'il considérait comme l'initiateur d'une " avant-garde hot », à savoir d'un conceptualisme politique engagé.

- Sabrina D. Marques. En cherchant à définir la notion d'avant-garde, Ernesto de Sousa a engagé une guerre. Une " guerre contre le spectaculaire indépendant de la vie elle-même ». Une " guerre contre l'illusion et la passivité du spectateur ». Une " guerre contre le consumérisme suprême et l'individualité de l'œuvre d'art ${ }^{29}$ ». Politiquement, il s'agit d'un espace de fusion entre l'artiste et l'individu. Le patronyme d'un auteur n'est donc pas un emblème de la société de consommation, son art n'est pas un produit, digestible en tant que tel. Au contraire, Ernesto de Sousa définit l'avant-garde comme un outil d'analyse, un processus ouvert ; « recherche de l'œuvre d'art comme processus, relativement aux mutations sociales et individuelles ${ }^{30}$ ».

Ce principe d'effacement de l'auteur, permettant au spectateur de vivre une expérience émancipatrice, $s$ 'incarne particulièrement dans des œuvres ouvertes telles que Almada, um nome de guerra (fig. 6a-b). Cette œuvre multidisciplinaire propose un « cinéma élargi », combinant des matériaux filmiques, graphiques, poétiques, musicaux, picturaux et intégrant l'expérience artistique dans le champ des agitations réflexives et imprévisibles des perceptions synesthétiques.

- Raquel Schefer. À l'égard de la vocation collective présente dans l'œuvre d'Ernesto de Sousa, particulièrement manifeste lors de la création de la Cooperativa do Espectador, puis lors d'expériences collectivistes menées pendant et après la période révolutionnaire,

6b. Carlos Gentil-Homem et Ernesto de Sousa, affiche pour Almada, um nome de guerra, 1971, CEMES

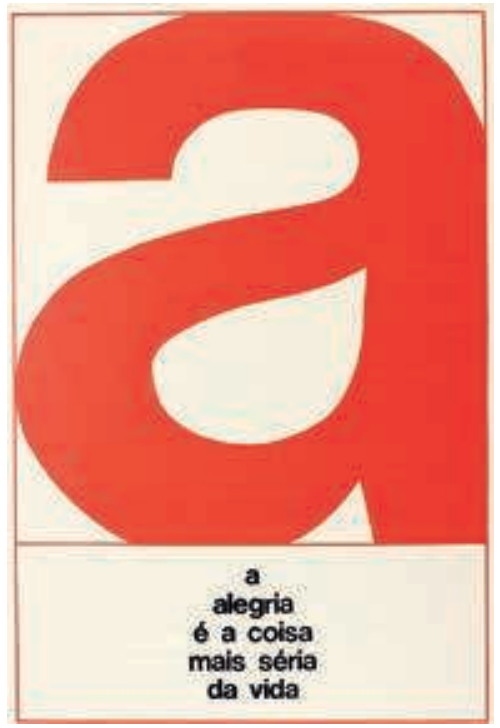


pensez-vous que la production d'Ernesto de Sousa, ses méthodologies et ses modes opératoires seraient en mesure de remettre en cause certaines catégories modernes, telles que les notions « d'auteur » et « d'œuvre d'art », encourageant ainsi la révision des relations entre l'art et la société dans le contexte portugais?

- Sabrina D. Marques. Il me paraît essentiel de souligner l'intérêt qu'Ernesto de Sousa a tout de suite porté à l'art populaire, qu'il préférait nommer « art d'expression naïve ». Dans le contexte de l'époque, il s'agissait d'une préoccupation inédite dans le domaine de la recherche esthétique, mais qui comportait son revers : une attention accrue aux conséquences de la globalisation avec l'arrivée de nouvelles références artistiques.

Autrement dit, le mouvement avec lequel Ernesto de Sousa comptait sauvegarder l'art des effets de la standardisation était double. Il y avait, d'un côté, une société portugaise à l'étude, avide de liberté par l'expression d'un art " du peuple », hors des circuits conventionnels, de l'argent, des marchés, des galeries, de l'académie et de la formation artistique et, de l'autre, les influences les plus modernes de la déconstruction critique que l'artiste importait de ses voyages. Ernesto de Sousa conserve spécifiquement la redéfinition des statuts d'artiste et de spectateur du groupe Fluxus qui écrit, dans I'un de ses manifestes (datant de 1965), que « [l'artiste] doit démontrer que tout peut se substituer à l'art et que tout le monde peut le faire. [...] [L'art] devrait être illimité, [...] et devrait finalement être produit par tous ${ }^{31}$. »

- Mickaël Robert-Gonçalves. Évidemment, la coopérative du Spectateur est un élément crucial pour comprendre la reconfiguration du cinéma portugais dans les années 1960 (fig. 7). Selon moi, elle a fusionné la relation du cinéphile avec celle du créateur, plus encore que ne le fait le cinéma français. En France, on est beaucoup revenu sur le rôle de la Cinémathèque française, dont l'origine est le ciné-club créé par Henri Langlois (qui, par ailleurs, portait le même nom que celui d'Ernesto de Sousa, "Le cercle du Cinéma »). François Truffaut, Jean-Luc Godard ${ }^{32}$, Claude Chabrol, Agnès Varda et les autres y incarnent la première génération de cinéphiles-critiques-cinéastes. Là où Ernesto de Sousa semble aller plus loin, c'est qu'il cherche également à redéfinir la notion d'auteur qui, en revanche, est restée au centre de la pratique cinématographique française (la fameuse « politique des auteurs »). La dernière période du nouveau cinéma portugais (avec notamment le Centre portugais du cinéma, vaste coopérative qui orchestre la production portugaise), puis le cinéma militant continuent de faire perdurer cet idéal coopératif initié Ernesto de Sousa. Cependant, à la fin des années 1970, I'auteur semble regagner sa place maîtresse, à l'image de Manoel de Oliveira qui devient le cinéaste portugais le plus reconnu, ou de João César Monteiro $^{33}$. L'influence des concepts défendus par le groupe Fluxus ${ }^{34}$, dont Ernesto de Sousa était proche dès la fin des années 1960, explique peut-être sa méfiance à l'égard de toute forme de frontière entre l'artiste et le public, l'art et la vie. Ce positionnement théorique est encore plus notable dans les années 1970, lorsqu'il organise « Alternativa zero »: Ernesto de Sousa s'est éloigné du cinéma, qui resterait un art où l'auteur est roi, pour promouvoir la vidéo et la performance, dans lesquelles l'expérience du public prime.

- Isabel Nogueira. Ernesto de Sousa considérait les artistes comme des « opérateurs esthétiques ». Il développait avec eux une relation de producteurs et de réalisateurs d'art. Par ailleurs, ses « actions » véhiculaient toujours les valeurs du collectif, de la festa, de la participation, de la politisation et du partage ${ }^{35}$. D'une manière générale, les événements collectifs ont marqué les années 1970 à travers des formes d'exposition multiples, menés par divers groupes, comme le grupo Acre (1874-1977), le grupo Puzzle (1976-1981), ou le Círculo de Artes Plásticas de Coimbra (1958), ou encore les « Encontros internacionais de arte » (1974-1977), 
lancés par Egídio Álvaro. Le collectif est donc une façon d'être ensemble caractéristique des années 1970, plus particulièrement dans la période qui suivit la révolution des CEillets. On peut d'ailleurs souligner le travail d'autres protagonistes, contemporains d'Ernesto de Sousa. Les expositions collectives ont marqué les années 1970 et les années 1980, de façon moins significative. Nous commençons enfin à écrire l'histoire de l'art portugais de cette période, du fait de la compréhension de certaines œuvres d'une justesse sans équivoque, dont la plupart s'inscrivent dans la continuité des actions d'Ernesto de Sousa. Les supports perpétuellement enchevêtrés et réinventés offrent une source d'expérimentation sans précédent. Mais il faut également signaler une autre caractéristique de l'art portugais de cette période : la jonction entre l'expérimentalisme, présent dans le mouvement néo-avant-gardiste international et l'art politisé, revendicatif, dénonciateur de la période révolutionnaire. C'est le cas notamment de certains films et collages d'Ana Hatherly. Personne n'avait encore véritablement étudié l'art portugais de cette époque, ce qui s'est fort heureusement produit au cours de ces dix dernières années.

- Raquel Schefer. L'œuvre d'Ernesto de Sousa a-t-elle une influence déterminante sur votre travail ? Si oui, à quel niveau?

- Isabel Nogueira. Indubitablement. J'ai commencé à m'intéresser à l'art portugais des années 1970 à partir de l'exposition "Alternativa zero ", puis à celui de la seconde moitié du XXe siècle jusqu'à nos jours. En 2007, mon premier livre revenait précisément sur cette exposition et sur les problématiques conceptuelles et historiques qu'elle soulevait. «Alternativa zero » marque une sorte d'apogée de l'avant-garde au Portugal. Elle offre une large visibilité à de nombreux artistes et préfigure le mouvement post-moderne. À partir de cette exposition, j'ai effectivement commencé à m'intéresser à Ernesto de Sousa et, par conséquent, à l'art portugais de la seconde moitié du XXe siècle. En 2021, mon dernier livre intitulé História da arte em Portugal: do Marcelismo ao final do século XX a été publié aux éditions Book Builders.

- Sabrina D. Marques. À l'ère d'internet, le flux continu d'images alimenté par des millions d'utilisateurs semble rendre obsolète la notion d'auteur. Les fantasmes de plusieurs décennies de recherches esthétiques s'accomplissent vraisemblablement à l'endroit du numérique, dont Ernesto de Sousa était certainement un précurseur. Les prédictions de Roland Barthes concernant une démocratisation de l'accès aux œuvres d'art grâce à la technique sont aujourd'hui célébrées en ligne. Les artistes du mail art se réunissent à travers un réseau international, s'inspirant des bases performatives du «spectateur émancipé », figure centrale de l'anti-cinéma qu'Ernesto de Sousa avait lui-même emprunté à Marcel Broodthaers. Avec les nouvelles possibilités du numérique, les supports de reproduction ne sont plus limités au corps 

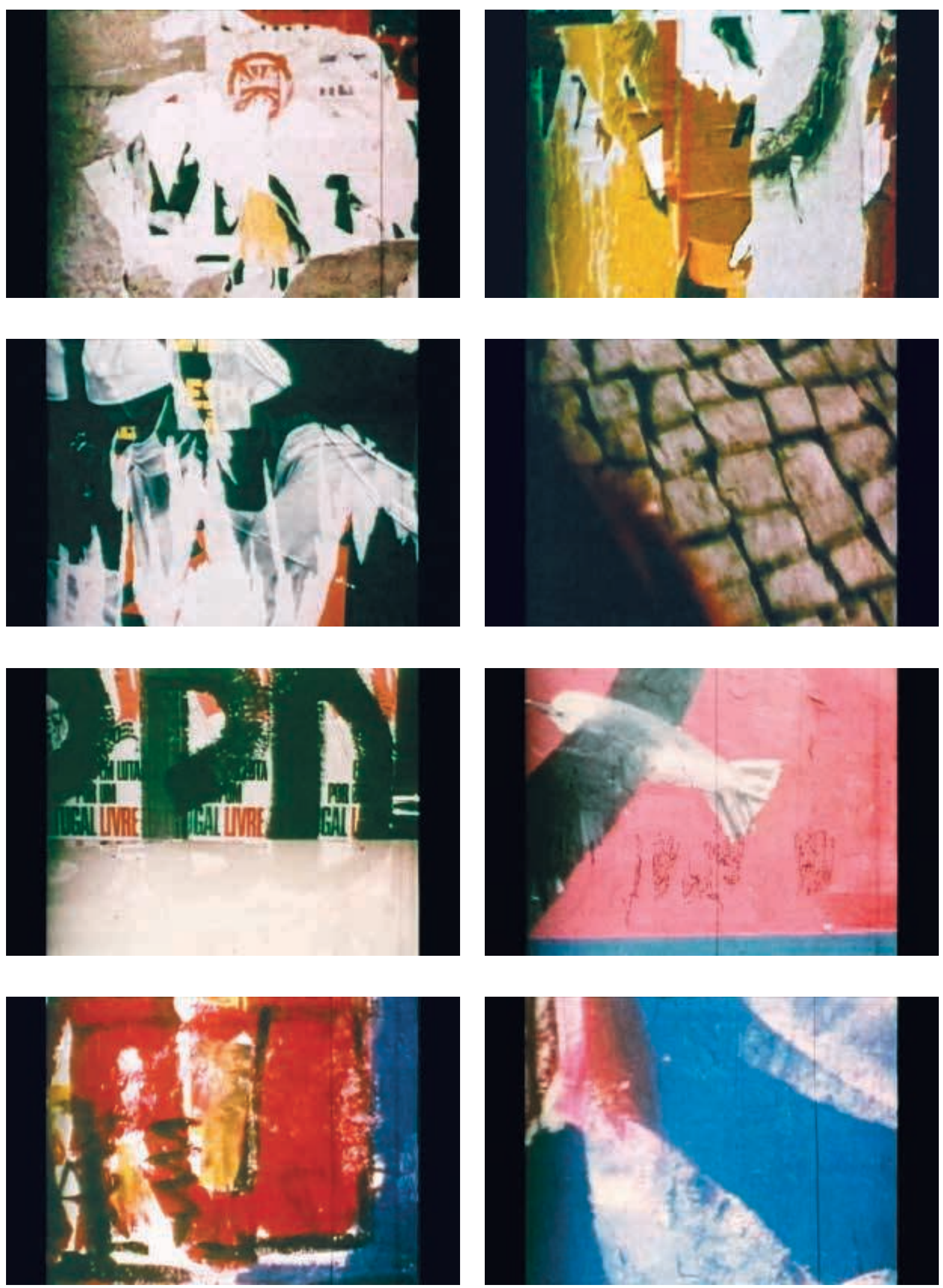
physique d'un objet. Cette dématérialisation est un héritage de la critique opérée par le happening contre la surconsommation et le "monde des choses".

L'étude des rapports entre l'art et l'espace public nous permet de redécouvrir le caractère expérimental de l'œuvre d'Ernesto de Sousa, tout en réactivant son " point de vue critique » toujours vibrant d'actualité.

- Mickaël Robert-Gonçalves. C'est surtout I'héritage théorique d'Ernesto de Sousa qui a eu un impact sur mon travail de recherche. Même s'il était naturel d'intégrer d'autres pratiques artistiques que le cinéma dans mes analyses, étant donné que les années 1970 correspondent à une période d'expérimen-

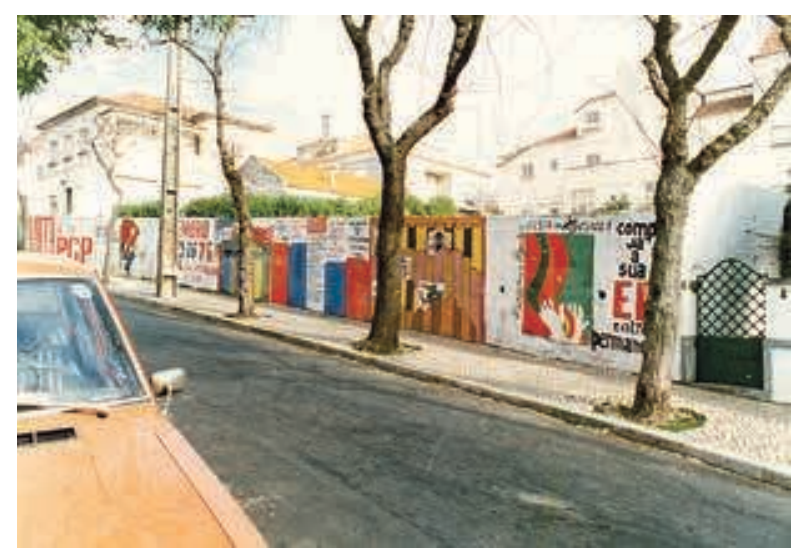

9. Fresques murales réalisées pendant la révolution des CEillets, en 1974, Oeiras, Santo Amaro, photographie issue des collections de l'Universidade de Coimbra, Centro de documentação 25 de abril, inv. $n^{\circ} 0100$. tations et de débats pour tous les arts, la place unique qu'occupe, par exemple, son projet Almada, um nome de guerra comme film-manifeste, puis ses écrits, m'ont permis de saisir la nature complexe des liens entre artistes, créations et, notamment, positionnements politiques par rapport à l'événement. Logiquement, dans ma thèse, je fais un pas de côté par rapport au cinéma documentaire militant pour aller vers les images les plus saisissantes de la révolution des CEillets, celles montées par l'artiste Ana Hatherly dans son film Revolução (fig. 8). Palimpseste de couleurs et de textures, scintillements de mots et de voix d'une révolution en cours, Revolução offre, selon moi, la mise en avant de l'image comme outil de la lutte. Le film est composé d'images - en ce sens, il déploie autant qu'il documente une part de l'iconographie de la révolution portugaise de 1974-1975 (fig. 9) -, mais se revendique, par sa fureur sonore, sa brièveté, comme un cri qu'Ernesto de Sousa interprète ainsi : « [l]e cri (l'action politique) et l'écriture, représentants du mot, se situent ainsi dans le pôle de l'ordre et du désordre, de la vie et de la mort. Être politique tient à l'un et à l'autre ${ }^{36}$. »C'est une lecture que je partage et qui alimente encore tout un pan de mon travail de recherche.

Les propos de Tiago Afonso, António Contador, Sabrina D. Marques, Isabel Nogueira et les questions de Raquel Schefer ont été traduits du portugais par Thomas Resendes. 


\section{Tiago Afonso}

Né à Porto en 1978, Tiago Afonso a étudié la photographie, la vidéo, le cinéma (cinéma documentaire, cinéma direct) et l'art contemporain. Il a dirigé des dizaines des films, et enseigné la réalisation et l'édition, notamment à l'Universidade Lusófona do Porto et dans d'autres établissement de formation intensive. Il a été premier assistant dans les films de Paulo Rocha, Saguenail, Edgar Pêra, Amarante Abramovici entre autres. Il a programmé dans plusieurs festivals et a été co-directeur du Doclisboa en 2015. Il a travaillé avec plusieurs compagnies de théâtre et groupes de musique. Ses films ont été programmés dans des festivals en France, au Portugal, au Brésil, en Espagne, en Italie, entre autres.

\section{António Contador}

António Contador vit et travaille à Paris. Après avoir fait des études en sociologie à I'Instituto universitário de Lisboa, il a obtenu son doctorat à I'université Paris 1 - Panthéon-Sorbonne (Arts visuels, esthétiques et sciences de l'art). Il a publié des articles et des ouvrages sur la question de la blackness au Portugal et, notamment, sur la culture de la jeunesse noire, ainsi que sur le thème des limites de I'art, avec un intérêt particulier pour certains gestes et performances artistiques liminaux. En tant qu'artiste visuel et performeur, il expose régulièrement depuis 2010 dans des lieux comme le palais de Tokyo, la fondation Ricard, la fondation Gulbenkian à Paris, le Wiels à Bruxelles, la Fundação de Serralves à Porto, Gasworks à Londres, ou le museu da República à Rio de Janeiro.

\section{Sabrina D. Marques}

Sabrina D. Marques (Paris, 1988) travaille dans la production, la recherche et la programmation des arts visuels, à Lisbonne. Dans le cadre de ses activités artistiques, elle travaille avec la vidéo, le film, la photographie, la performance et le textile. Elle est diplômée en sciences de la communication, spécialisée en cinéma, et doctorante en histoire de l'art contemporain, cinéma et photographie à I'Universidade Nova de Lisboa, où elle fait des recherches sur la photographie et la mémoire.

\section{Isabel Nogueira}

Isabel Nogueira est une historienne de I'art contemporain, essayiste et professeure. Après avoir obtenu son doctorat à I'Universidade de Lisboa (science de l'art), elle effectue un postdoctorat en histoire et théorie de l'art contemporain et théorie de l'image (Universidade de Coimbra / université Paris 1-Panthéon-Sorbonne). Elle est chercheuse au Centro de investigação e de estudos em belas-artes (CIEBA, faculdade de BelasArtes da Universidade de Lisboa), professeure à la
Sociedade nacional de belas artes, à Lisbonne, et rédactrice en chef d'Arte e Cultura Visual (CIEBA). Parmi ses publications récentes : L'image dans le cadre du désir : transitivité dans la peinture, la photographie et le cinéma (Paris, L'Harmattan, 2018), Teorias da arte: do modernismo à actualidade (Silveira, Book Builders, 2019), Como pode 'isto' ser arte? Breve ensaio sobre crítica de arte e juízo de gosto (Ribeirã, Húmus, 2020), História da arte em Portugal: do Marcelismo ao final do século XX (Silveira, Book Builders, 2021).

\section{Mickaël Robert-Gonçalves}

Mickaël Robert-Gonçalves est docteur en études cinématographiques et audiovisuelles de l'université Paris 3 - Sorbonne-Nouvelle. Il est actuellement chargé de cours dans plusieurs universités et écoles, travaille pour la Cinémathèque française et a publié plusieurs articles sur l'histoire du cinéma portugais et les liens entre cinéma et politique.

\section{Raquel Schefer}

Docteur en Études cinématographiques et audiovisuelles (université Paris 3 - Sorbonne-Nouvelle), Raquel Schefer est chercheuse, cinéaste, programmatrice et enseignante à l'université Paris 3 - Sorbonne-Nouvelle. Elle a publié l'ouvrage EI autorretrato en el documental (Buenos Aires, Catálogos, 2008), et de nombreux chapitres d'ouvrages et articles. Parallèlement à ses activités en tant que rédactrice en chef de la revue de théorie et d'esthétique du cinéma La Furia Umana, elle mène actuellement une recherche post-doctorale à I'Université du Western Cape et au Centro de Estudos Comparatistas de l'Universidade de Lisboa, où elle est directrice du projet « Visual Culture, Migration, Globalization and Descolonization ", et à I'Istituto de História contemporânea (Universidade Nova de Lisboa), où elle coordonne I'Oficina de História e Imagem.

\section{NOTES}

1. Selon l'expression de Gene Youngblood, Expanded Cinema, New York, E. P. Dutton \& Co., 1970. Ernesto de Sousa, Almada, um nome de guerra, techniques mixtes avec une musique orginale composée par Jorge Peixinho, 1969-1972. Film (35 mm et $16 \mathrm{~mm}$; noir et blanc, et couleur ; muet) et projection de diapositives (noir et blanc, et couleur) ; son (musique enregistrée, avec de possibles interventions musicales, lectures de textes et ajouts d'autres éléments sonores) ; matériel graphique imprimé (affiches et autocollants). Durée variable : 20 à 40 minutes (version courte) ou 240 minutes (version complète).

2. Isabel Nogueira, "Os 40 anos da "Documenta 5" (1972): reflexo e reflexões sobre a arte portuguesa 
dos anos 70 », Artecapital, 10 décembre 2012 [URL : artecapital.art/perspetiva-147-isabel-nogueira-os -40-anos-da-documenta-5-1972-reflexo-e-reflexoes -sobre-a-arte-portuguesa-dos-anos-70], traduction de l'auteure.

3. « [O]peradores estéticos », selon l'expression d'Ernesto de Sousa, "Ana Hatherly e a difícil responsabilidade da desordem ", Colóquio-Artes, n 36, mars 1978 [URL : ernestodesousa.com/bibliografia/ana -hatherly-e-a-dificil-responsabilidade-da-desordem], traduction de l'auteure.

4. «Que ele é talvez o homem mais extraordinário que me foi dado conhecer, não adianta muito ao caso. Mas que nomeando-o descubro um NOME DE GUERRA para tudo ou muito do que mais me interessa fazer ", Ernesto de Sousa, " Ainda não filmei as varinas todas: o antifilme Almada, um nome de guerra ", Diário de Lisboa, 16 avril 1970, cité dans "Almada, um nome de guerra", "Projectos ", sur le site du Centro de estudos multidisciplinares Ernesto de Sousa [URL. : https://www.ernestodesousa.com/projectos/almada-um-nome-de-guerra], traduction de l'auteure.

5. Nogueira, 2012, cité n. 2, traduction de l'auteure.

6. Peter Bürger, Théorie de l'avant-garde, Paris, Saggio Casino, 2013.

7. Ernesto de Sousa, "Anticinema ", texte inédit, vers 1969, reproduit sur le site du CEMES [URL : ernestodesousa.com/bibliografia/anticinema], traduction de l'auteure.

8. « ([P]eço ao leitor que complete as frases como muito bem entender. E a pontuação também / isto não é modéstia é convite a uma outraforma de trabaIho colectivo / leitura operativa / e a culpa é nossa se o leitor / preguiçoso) [...] UMA ARTE COLECTIVA UMA ARTE DE PARTICIPAÇ̃̃O / ARTE ABERTA OU ANTI-ARTE (é o mesmo) / por exemplo o cinema / destruir reconstruir?) / destruir o cinema / não como acto gratuito / nem como provocação ", ibidem.

9. « [D]emonstrar a importância menor do objecto de arte, face aos sujeitos envolvidos pela atividade estética, face ao PROCESSO ESTÉTICO ", Ernesto de Sousa, Alternativa zero: tendências polémicas na arte portuguesa contemporânea, cat. exp. (Lisbonne, Galeria nacional de arte moderna), Lisbonne, Secretaria de estado da cultura, 1977, traduction de l'auteure.

10. Voir Isabel Nogueira, "Os 50 anos do Encontro no Guincho (1969): experimentalismo, poética, tempo ", Convocarte, no 9, 2020, p. 245-254.

11. " Do Vazio à Pró Vocação ", exposition à Lisbonne, Sociedade nacional de belas-artes, juillet-août 1972, intégrée à l'exposition de l'AICA de 1972.

12. "O estado zero: encontro com Joseph Beuys", República, 28 décembre 1972, republié dans Ser Moderno... em Portugal, Lisbonne, Assírio \& Alvim, 1998.

13. Isabel Nogueira, Do pós-modernismo à exposição Alternativa zero, Lisbonne, Nova Vega, 2007 ; Alternativa zero (1977): o reafirmar da possibilidade de criação, Coimbra, CESIS20 / Universidade de Coimbra, 2008.

14. Voir "Almada Negreiros e Ernesto de Sousa: o "ser moderno em Portugal" », dans António Pedro Pita et Luís
Trindade (dir.), Transformações estruturais do campo cultural português (1900-1950), Coimbra, Ariadne Editora / Universidade de Coimbra, 2005, p. 281-295.

15. Mickaël Robert-Gonçalves, "Cinéma portugais en Révolution. 1974-1982 : genèse, enjeux, perspectives ", thèse de doctorat, université Paris 3 - SorbonneNouvelle, 2018. Cette thèse revient sur l'histoire politique du cinéma portugais depuis les années 1950-1960 jusqu'au début des années 1980, en se concentrant plus particulièrement sur les modalités esthétiques selon lesquelles les artistes se sont engagés dans le processus révolutionnaire.

16. Extrait d'un entretien avec Alberto Seixas Santos réalisé en février 2011 à Lisbonne, cité dans RobertGonçalves, 2018, cité n. 17, p. 54.

17. La Cooperativa do Espectador ("Coopérative du spectateur »), créée sous l'impulsion d'Ernesto de Sousa, du ciné-club et de la revue Imagem, a permis de lever des fonds auprès des spectateurs pour produire des films, notamment Dom Roberto. Le projet était en effet pensé pour être durable et favoriser la création cinématographique de jeunes cinéastes, notamment dans le court-métrage. L'échec en billetterie de Dom Roberto eut raison des ambitions initiales.

18. «Pude então estudar com rigor a evolução das vanguardas, ou melhor, a vanguarda; porque há só uma. » Ernesto de Sousa, "Alternativa zero: uma criação consciente de situações ", dans Colóquio / Artes, n 34, 1977, p. 45-53.

19. Idem, « Há tanta gente, Mariana! », Opção, n 123, 1978 , p. 42.

20. Sousa, 1998, cité n. 14, p. 122.

21. Ibidem, p. 67.

22. Chroniques de l'art vivant, $\mathrm{n}^{\circ}$ 32, août-septembre 1972.

23. Maurice Blanchot, "Le musée, l'art et le temps", L'Amitié, Paris, Gallimard, 1971, chap. II, p. 35.

24. À propos de la visite d'Ernesto de Sousa à « Documenta 5 ", voir Filipa Ramos et António Contador, "Revisitando Ernesto de Sousa em Kassel », dans Luísa Seixas, Lígia Afonso, Gabriela Vaz Pinheiro (dir.), Laboratório de Curadoria, Guimarães, Capital da Cultura, 2012, p. 369-392.

25. Ilda Teresa Castro, « António Reis e o espaço de um lugar - sintomatologias de Jaime ", dans Paulo Cunha et Sérgio Dias Branco (dir.), Atas do III encontro anual da Associação de investigadores da imagem em movimento, Lisbonne, AIM, 2014, p. 28.

26. Ernesto de Sousa, "Vanguarda e Empenhamento ", s.l., 1974, publié sur le site du CEMES [URL : ernestodesousa.com/bibliografia/vanguarda-e-empenhamento].

27. Voir Sousa, 1977, cité n. 20, p. 51.

28. Voir idem, "A ordem o acaso e a festa ", Vida Mundial, n 1852, 1975, p. 43-44.

29. «Combate ao espectáculo (independente da própria vida) e à sublimação por via estética da memória colectiva. Combate a todo o ilusionismo e à passividade espectatorial. Combate ao consumismo, e, em particular, ao consumismo sublime (propriedade 
individual da obra de arte). » Ernesto de Sousa, cité par João Fernandes, "Perspectiva: Alternativa zero, vinte anos depois... ", Perspectiva: Alternativa zero, Porto, Fundação de Serralves, 1997.

30. «Procura da obra de arte como processo (e relativa a sujeitos em processo, sociedades em processo...) », ibidem.

31. " [The artist] must demonstrate that anything can substitute art and anyone can do it. [...] It [Art] must be unlimited, [...] and eventually produced by all. » George Maciunas, Fluxmanifesto on Fluxamusement, 1965.

32. Le parcours de Godard, en particulier, a beaucoup influencé les cinéastes portugais dans les années 1970. La constitution du groupe Dziga Vertov par Jean-Luc Godard et Jean-Pierre Gorin en 1968-1969 correspond à la volonté de faire "politiquement des films politiques ». En outre, ce tournant maoïste dans l'œuvre godardienne rejoint également l'idée d'un effacement de la place de l'auteur qu'Ernesto de Sousa a semblet-il également recherché.

33. João César Monteiro, connu pour son œuvre inclassable faite de films-poèmes et porté par le personnage qu'il incarne lui-même, João de Deus, a également participé au moment militant du cinéma portugais en réalisant le film Que farei eu com esta espada? ("Que ferai-je de cette épée? »), en 1975.

34. Même si des artistes relativement différents ont été proches du groupe Fluxus, tous ne partageaient pas les préceptes du mouvement. Cependant, d'une manière générale, Fluxus a eu une certaine influence dans la volonté de désacraliser l'art, de le mettre à la portée de tous, en valorisant le mode d'action direct et participatif, comme les happenings. La notion même d'œuvre d'art est remise en cause. L'artiste ne doit pas se cantonner à l'expression nombriliste de son identité, mais chercher à s'effacer au profit du collectif.

35. Voir Isabel Nogueira, "A performance como arte e festa: democratização, eventos colectivos e espaço público ", dans Ana Pais (dir.), Performance na esfera pública, Lisbonne, Orfeu Negro, 2017, p. 63-74.

36. «O grito (a acção política) e a escrita, vigários da palavra, situam-se assim no pólo da ordem e da desordem, da vida e da morte. Ser político, participa de ambos »dans Sousa, 1978, cité n. 3, p. 29, traduction de l'auteur. 